\title{
Early-stage lung cancer: a report from the recent ERS School Course
}

On February 8-10, we were given the privilege of hosting an external course on earlystage lung cancer, which was organised by the ERS School. The course took place in Strasbourg (France), and the co-organisers were Elisabeth Quoix (Head of the Department of Pulmonology, University Hospital of Strasbourg; current Chair of Group 1.05 Thoracic Oncology) and Jean-Paul Sculier (Head of Thoracic Oncology at the Jules Bordet Cancer Center in Brussels; former Chair of Group 1.05).

There was a relatively low number of attendees at the course when compared with other external courses. However, the choice of the topic was quite specialised, since most respiratory physicians actually encounter the situation of inoperable and/or advanced stage disease, rather than early-stage disease. This however meant that all the participants were highly motivated and contributed enthusiastically, making this a very interactive and successful venture.

The aims of this editorial are three-fold. First, we would like to discuss the content of the course. Secondly, we wish to discuss the choice of the review on "What's new in the epidemiology of lung cancer: the female aspect", which is published in this issue of Breathe and has been adapted from one of the presentations on the School Course. Finally, we feel the need for some more general considerations on lung health beyond the domain of oncology.

The course was presented by selected experts, who provided state of the art information on several fundamental questions. Epidemiology issues were addressed by Pascal Bofetta and Elisabeth Quoix, and included new insights into the disease and the recent evolution of epidemiology in general. AnnePascale Meert gave stimulating lectures on pathology with an emphasis on carcinoma in situ and molecular biology. Vincent Ninane gave an overview on endoscopic detection and staging of lung cancer, and set criteria for the endoscopic treatment of carcinoma in situ. The discussion was continued with an insight into the remaining indications of surgical staging by Paul Van Schil, whereas Pascal Thomas debated on surgical treatment of very early lesions. In a dynamic and engaging manner, all three participating surgeons explained the basic principles of surgical treatment, consisting of anatomic resection combined with radical lymph node resection, and some alternatives such as segmental resection or bronchoplastic resection.

The innovative curative approach consists of the addition of neoadjuvant or adjuvant chemotherapy. These areas were examined by Jean-Paul Sculier and Virginie Westeel. While there seems to be a survival advantage for patients with stage II or III disease subjected to multimodality treatment, it is yet unclear which modality should be chosen. Neodjuvant therapy allows the monitoring of the effects of chemotherapy, but could increase surgical morbid mortality; adjuvant therapy is applicable to exhaustively staged patients, but could suffer from less than optimal compliance to treatment. Paul van Houtte gave very thoughtful lectures on the indications of radiation therapy. Some final comments addressed the controversial issue of post-treatment follow-up.

A time-trend analysis performed by Elisabeth Quoix showed that global results had not improved significantly for non-small cell lung cancer on the whole, but that there is a striking difference in prognosis when comparing different European countries. Five-year survival rates of all cancer patients taken together range $5-12 \%$, and less than one third are eligible for curative treatment. Conversely, the life expectancy of small cell lung cancer patients has significantly increased over the past 20 years.

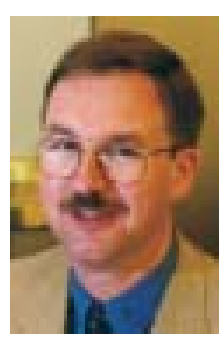

G. Massard ${ }^{1}$

E.A. Kochetkova²

1 Université Louis Pasteur and Service de Chirurgie Thoracique, Hôpitaux Universitaires de Strasbourg, Strasbourg, France.

2Vladivostok State Medical University and Far Eastern Medical Center, Vladivostok, Russian Federation.

\section{Correspondence:}

G. Massard

Université Louis Pasteur and Service de Chirurgie Thoracique Hôpitaux Universitaires de

Strasbourg

67091 Strasbourg

France

E-mail:

Gilbert.Massard@chru-strasbourg.fr 


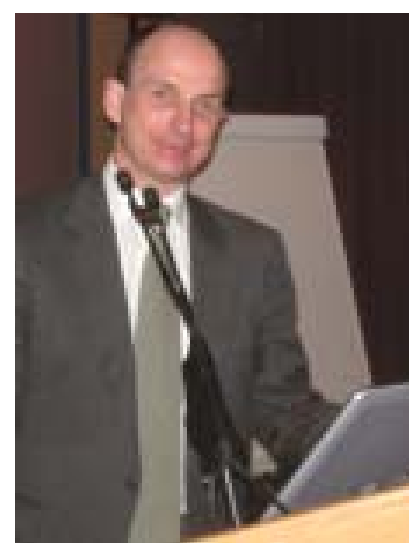

Vincent Ninane

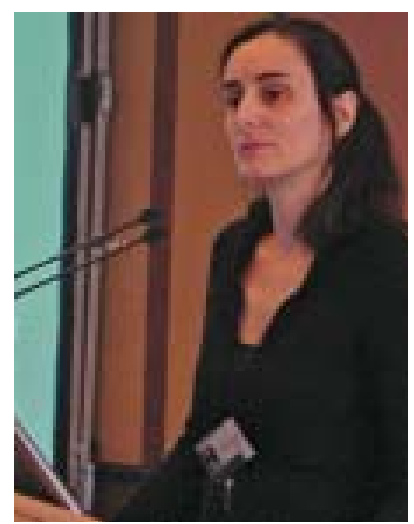

Virginie Westeel
In this issue of Breathe, the Editorial Board has chosen to publish an article on new data in the epidemiology of lung cancer, written by a well-recognised specialist in this topic, namely Elisabeth Quoix. Both co-authors of this editorial thoroughly endorse the choice of this paper. While the usefulness of technical information on treatment of lung cancer is limited to those who treat such patients in their everyday practice, this paper raises some very important issues for all colleagues dealing with lung diseases.

The first point to consider is that the article confirms a well-documented relationship between lung cancer and tobacco consumption. We would like to emphasise that not only active smoking but also passive smoking is dreadful. For several years now, lung cancer has been the most frequent cause of death by cancer in males. A new phenomenon is the dramatic increase in prevalence of lung cancer in females, and for almost 20 years, mortality due to lung cancer in females has surpassed mortality from breast cancer.

The second point is changing histology. There is a slight and constant move, replacing the leading position of squamous cell cancer with adenocarcinoma, which is explained by changing tobacco consumption.

The third and most alarming point is that, for several reasons, females are more sensitive to tobacco consumption, and that a far lesser quantity of cumulated exposure to smoking is required for efficient carcinogenesis in females. The reasons are unclear, but there could be a genetic predisposition and potential hormonal factors.

Last but not least, a distressing observation made by epidemiologists is that prevalence of lung cancer is increasing in non-smokers; the reasons for this recent phenomenon are as yet unclear.

These observations lead us to more general considerations, going beyond the domain of oncology. The best medicine is the one that prevents people from getting sick. It is well established that the most life-threatening lung diseases, i.e. lung cancer and chronic obstructive pulmonary disease (COPD), are obviously related to tobacco consumption. The fact that this editorial is co-authored by an Eastern and a Western expert should highlight the pan-
European endemic spread of smoking-related lung disease.

In 1990, lung cancer was rated as the 3rd highest cause of death after ischaemic heart disease and cerebrovascular disease across the whole of Europe. By 2010, the first three causes of death are expected to stay the same. However, COPD is expected to rise from 5 th to 4th in western European countries, and from 8th to place 4th in eastern European countries. With that said, in 2010, the four leading causes of death in the Euro-Asian areas of the globe will be related to tobacco consumption! In the large European area defined by the World Health Organization (WHO), which includes Russia beyond the Ural and the Central Asian Republics, $20 \%$ of all cancer deaths are attributed to lung cancer. Lung cancer is definitely a devastating disease: while the annual incidence of new diagnoses made in Europe is $\sim 46$ per 100,000, the European annual average for mortality is close to 38 per 100,000 . Approximately $90 \%$ of lung cancer cases are caused by smoking and the WHO has officially declared second-hand smoke as carcinogenic to humans. COPD causes an average of 50 deaths per 100,000 in males, and 20 per 100,000 in females in Europe (as defined by the $\mathrm{WHO}$ ).

Beyond these tremendous death rates, there is a considerable economic burden caused by smoking-related chronic lung disease. Whereas unfortunately the majority of patients suffering from lung cancer eventually die from their disease, COPD causes long-term invalidity, which economists translate into a loss of 41,300 workdays per 100,000 population in the European Union.

The final message of this editorial is a wish for the future. Our populations need excellent preventive medicine, especially since smokingrelated lung diseases are incurable. The fight against tobacco consumption, in which the European Respiratory Society is very actively involved, offers a real chance to decrease the leading causes of death and disability in European countries, namely lung cancer and COPD, but also cardiovascular disease and stroke.

The material from the presentations given at the School Course are available on the ERS website at www.ersnet.org/elearning 